\title{
Verschobene Kräfteverhältnisse
}

\author{
Das Deutsche Institut für Urbanistik hat im Rahmen einer Sondierungsstudie für \\ das Bundesministerium für Bildung und Forschung sozial-ökologische Probleme \\ und Chancen untersucht, die aus der zunehmenden Privatisierung und Liberali- \\ sierung bisher öffentlicher Aufgabenbereiche erwachsen können (1). Es zeigt \\ sich, dass sich die Rolle und auch das Selbstverständnis von Kommunen erheb- \\ lich verändern wird. Für die Bürger als Verbraucher können daraus neve Chan- \\ cen erwachsen, wenn Markttransparenz und die Einhaltung ökologischer Rah- \\ menbedingungen gewährleistet bleiben.
}

$\mathrm{U}$ Von Jens Libbe $m$ die künftigen kommunalen Steuerungsmöglichkeiten analysieren zu können, muss zwischen dem Wettbewerb im Markt und dem Wettbewerb um den Markt unterschieden werden. Dies legen der aktuelle Stand der Diskussion, aber auch die real ablaufenden Prozesse in einzelnen Sektoren kommunaler Daseinsvorsorge nahe. In diesen Wettbewerbsmodellen werden bislang integrierte Prozessketten aufgesplittet. Die Stufen der Erzeugung - etwa von Strom - bzw. Gewinnung - etwa von Wasser -, des Transports und der Verteilung bzw. Versorgung werden separat betrachtet. Das vormals integrierte Monopol wird letztlich auf ein natürliches Netzmonopol reduziert.

Die umfassende Marktöffnung von Ver- und Entsorgungsnetzen und die Einbeziehung privater Unternehmen in die Erstellung und Erbringung von Leistungen der Daseinsvorsorge bedeutet keineswegs den Wegfall hoheitlicher Aufgaben in diesen Bereichen. Vielmehr handelt es sich um einen aufwändigen Wandel von öffentlichen Aufgaben und Steuerungsformen. So setzt die Liberalisierung einerseits neue Formen staatlicher Marktregulierung voraus, um etwa einen funktionsfähigen und diskriminierungsfreien Wettbewerb zu ermöglichen. Andererseits bestehen wichtige öffentliche Interessen und Belange trotz der Liberalisierung fort. Ein erheblicher Regulierungsbedarf besteht auch künftig in Hinblick auf die direkten und indirekten ökologischen Folgelasten.

\section{- Die zukünftige umweltpolitische Rolle der Kommunen}

$\mathrm{Zu}$ fragen ist allerdings, welche umweltpolitischen Ziele und Maßnahmen künftig noch oder verstärkt Aufgabe der Kommunen sein sollten bzw. kön- nen und welche von den übergeordneten Ebenen gewährleistet werden müssen. Generell gilt, dass im föderativen Staat für die Entscheidung zur Erfüllung einer Aufgabe diejenige Ebene zuständig sein sollte, die alle Nutznießer und Kostenträger umfasst (Prinzip der fiskalischen Äquivalenz). Maßgeblich für die geeignete Zuordnung von umweltpolitischen Entscheidungskompetenzen sollte allerdings auch die räumliche Ausdehnung der jeweiligen Umweltbelastung sein (territoriale Äquivalenz).

Nun zeigt etwa das Beispiel des Klimaschutzes, dass die Emissionsminderungsziele nur dann erreicht werden können, wenn notwendige internationale und nationalstaatliche Regulierungsformen um Steuerungsmaßnahmen auf untergeordneten Ebenen ergänzt werden. Dabei ist vermutlich weniger an steuerliche oder Recht setzende Maßnahmen zu denken, die Aufgabe der Europäischen Union und des Bundes sind, als an die Förderung der Kooperation unterschiedlicher Akteure oder das Freisetzen von Innovationskräften auf dezentraler Ebene.

Gerade im Wettbewerb im Markt dürfte die Rolle der Kommunen künftig stärker in einer nachfrageorientierten Umweltpolitik liegen, etwa durch Social Marketing und Aufklärung. Unklar ist jedoch, wie effizient und effektiv nachfrageseitige Strategien im Vergleich zu angebotsorientierten Maßnahmen sind. So hat Öko-Strom gegenwärtig nur einen Marktanteil von einem Prozent. Es regiert der Preis, die Bürger sind nicht bereit, mehr zu zahlen. Liberalisierung wird gerade über reduzierte Preise für zum Beispiel Strom und Wasser propagiert. Die Frage ist, wie Anreize auf der Nachfrageseite gesetzt werden können und ob die Differenz zwischen Wissen und Handeln überwunden werden kann.
Veränderungen im Kräftedreieck Unternehmen - Kommune - Bürger

Die beiden identifizierten Wettbewerbsmodelle zeitigen nicht nur Auswirkungen auf die kommunalen Regulierungspotenziale, sondern auch auf bürgerschaftliche Kontrollmöglichkeiten und dürften Veränderungen im Verhältnis zwischen Bürgern und Kommune nach sich ziehen.

Im Wettbewerb um den einzelnen Kunden werden vermutlich Kosten- und Qualitätsaspekte, die direkt vom Kunden zur Kaufentscheidung herangezogen werden, sowie das Marketing eine Rolle spielen. Die Kommunen bzw. ihre Betriebe sind hier nur noch ein Wettbewerber unter vielen, der sich über Preise, Qualität und Service im Wettbewerb behaupten muss. Damit verlieren die Kommunen und ihre Betriebe ihren Sonderstatus als primäre Ansprechpartner gegenüber ihren Bürgern. Umgekehrt werden die Bürger für die Kommune zu Kunden, die es im Wettbewerb gegenüber privaten Firmen zu halten bzw. zu gewinnen gilt.

Im Wettbewerb um Konzessionen fungiert die Kommune als Auftraggeber gegenüber privaten Unternehmen, die sich in einer Ausschreibung um die Erbringung einer definierten Leistung beworben haben. Eine qualitativ neue politische Rolle könnte die Kommune einnehmen, indem sie den Prozess der Konkretisierung und Formulierung der zu vergebenden Konzession moderiert und die Wünsche und Bedürfnisse der Bürger/ Nutzer mit anderen stadtentwicklungspolitischen Vorhaben abgleicht. Die so in einem partizipativen, kooperativen Prozess gewonnenen Zielvorgaben schreibt die Kommune in die Ausschreibung der Konzessionsverträge hinein. Die Kommune nähme damit die Rolle einer intermediären Institution zwischen ihren Bürgern und den privaten Unternehmen ein.

\section{Gefahren und Chancen}

$\mathrm{Zu}$ einer Gefährdung für die Kommunen in einem eher traditionellen Verständnis könnten sich die skizzierten Entwicklungen verdichten, wenn es zu einer Entkommunalisierung im Sinne einer Privatisierung in zweifacher Hinsicht käme: sowohl auf der unternehmerisch-wirtschaftlichen Seite (Privatisierung der Leistungserbringung) als auch im zivilgesellschaftlichen Bereich (Privatisierung der demokratischen Aushandlungsprozesse). Traditionelle Vorstellungen dessen, was Kommune ist und ausmacht, nämlich kommunale Selbstverwaltung und kommunale Daseinsvorsorge, würden gegebenenfalls obsolet. 
Zugleich liegen in den oben skizzierten Entwicklungen Potenziale für zukünftige neue Rollen und Aufgaben der Kommunen jenseits einer zunehmenden Normierung des kommunalen Handelns durch staatliche Gesetzgebung und einer Trivialisierung der Kommunalpolitik. Schließlich birgt der institutionelle Wandel in Kommunen Chancen für eine Re-Politisierung der Kommunen und die Neuaushandlung dessen, was Daseinsvorsorge und Gemeinwohlbelange beinhalten sollten.

Aus der Perspektive der Kunden jedoch steigen durch die Liberalisierung und Privatisierung möglicherweise die Chancen einer direktdemokratischen Steuerung der Unternehmen im Sinne einer „Kundendemokratie“. Geht man von der Prämisse einer Kundenorientierung privater Unternehmen aus, so sollte sich - zumindest in der Theorie - durch die autonome und rationale Kaufentscheidung der einzelnen Kunden eine optimale Anpassung des Angebots an die Nachfrage einspielen. Praktisch kann jedoch nicht von einem ausgeglichenen Verhältnis von Angebotsund Nachfrage- bzw. Verbrauchermacht ausgegangen werden. Zum einen sind die Interessen der einzelnen Verbraucher in hohem Maße heterogen und damit nur bedingt als gebündelter Machtfaktor gegenüber den Anbietern einsetzbar. Zum zweiten bestehen auf der Nachfrageseite oftmals Informationsdefizite über die Qualität und Preise der Produkte, die nur mit einem hohen Kostenaufwand ausgeglichen werden können.

\section{- Neve Rolle von Verbraucherverbänden}

In diesem Zusammenhang könnte Verbraucherverbänden eine neu gewichtete Rolle zuwachsen. In Großbritannien etwa können die Kunden der privaten Wasserversorgungsunternehmen vermittelt über sogenannte consumer committees Einfluss auf die Gestaltung der Wasserversorgung in ihrem Versorgungsgebiet nehmen. Organisierte Interessenvertretungen der Kunden stehen damit neben anderen Institutionen, wie zum Beispiel der Regulierungsbehörde, unmittelbar in der öffentlichen Verhandlung über Qualität und Preise der Wasserversorgung. Die Bedeutung der Verbraucherverbände könnte demnach auch in Deutschland sowohl hinsichtlich der zu leistenden Informationsarbeit als auch bezogen auf die konkrete Einflussnahme auf (kommunal-)politische Verhandlungsprozesse und unternehmerische Entscheidungen steigen.

Zugleich wird das Management und der Umgang mit Informationen nicht nur zwischen Ver- und
Entsorgungsunternehmen und Kunden an Bedeutung zunehmen. Auch der Informationsfluss zwischen Kommune und formal oder materiell privaten Unternehmen dürfte sich verändern. Unklar ist bisher, wie diese Veränderung aussehen und welche Konsequenzen sie nach sich ziehen wird. Plausibel scheint die Befürchtung, dass es zu einer Abnahme des Informationsflusses vom privaten Unternehmen an die Kommune kommt, da im Wettbewerb nicht mehr alle unternehmensbezogenen Daten veröffentlicht werden, um potenzielle Wettbewerbsvorteile gegenüber Konkurrenten nicht preiszugeben. Damit verfügt die Kommune nicht mehr selbstverständlich über alle für die politische Debatte um die Stadt- oder Gemeindeentwicklung relevanten Daten und könnte diese auch der Öffentlichkeit nicht mehr zugänglich machen.

\section{- Fazit}

Insgesamt legen die Ergebnisse der Studie den Schluss nahe, dass die umwelt- und sozialpolitischen Konsequenzen der Privatisierung und Liberalisierung öffentlicher Aufgaben, sowie die sich in diesem Zusammenhang stellenden demokratietheoretischen Fragen, einer differenzierten Betrachtung bedürfen. In jedem Fall sollten die Kommunen diese stärker als bisher in Betrachtung ziehen, um etwa die Veränderungen ihrer eigenen Gestaltungsmöglichkeiten als Folge einer Privatisierungsmaßnahme besser beurteilen zu können.

\section{Anmerkung}

(1) Der Endbericht zur Sondierungsstudie „Liberalisierung und Privatisierung öffentlicher Aufgabenbereiche in Kommunen. Sozial-ökologische Problemlagen und Chancen für eine nachhaltige Entwicklung" kann im Internet unter http:// www.difu.de/archiv abgerufen werden. Die Studie wurde vom Autor gemeinsam mit Stephan Tomerius und Jan Trapp durchgeführt. Für das BMBF bildete die Studie eine Grundlage für den Schwerpunkt "Sozial-ökologische Regulation im Ver- und Entsorgungssektor (STRIVE) im Rahmen des Förderschwerpunkts "Sozial-ökologische Forschung".

\section{Der Autor}

Jens Libbe ist wissenschaftlicher Mitarbeiter am Deutschen Institut für Urbanistik (Difu), Arbeitsbereich Umwelt und Verkehr.

Kontakt: Difu, Straße des 17. Juni 112, 10623 Berlin. Tel. 030/ 39001-115, Fax -150, E-mail: libbe@difu.de

\section{Neuerscheinung:}

UnternehmensGrün Band 8

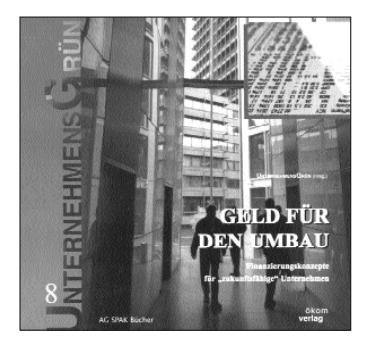

\section{Geld für den Umbau \\ Finanzierungskonzepte für \\ "zukunftsfähige" Unternehmen}

Für kleine und mittlere Unternehmen wird es immer schwieriger, die notwendige Kapitalbasis für ihre sozialen und ökologischen Innovationen zu organisieren. Ihnen bleibt oft nur die mühevolle Suche nach Eigenund Beteiligungskapital bei Investoren. UnternehmensGrün fasst daher entsprechende Finanzierungsmöglichkeiten zusammen. AutorInnen aus Politik und Bankenwelt diskutieren die verschiedenen Methoden von Eigen- und Fremdkapitallösungen, über Mitarbeitermodelle und grüne Beteiligungsgesellschaften bis hin zur grünen Börse. "Geld für den Umbau" zeichnet die konkrete Vision einer alternativen Börse. Soziales und ökologisches Engagement führt langfristig - neben guten Renditen - zum „Fairholder value".

Unternehmensgrün, Verband zur Förderung umweltgerechten Wirtschaftens (Hrsg.), Band 8, 12,80€, ökom Verlag, ISBN 3-928244-73-6.

Band 1

"Augen zu und durch?

Was kommt nach der Krise?" - Über den

konstruktiven Umgang mit der Rezession

Band 2

Erhalt durch Veränderung.

Perspektiven einer ökologischen und sozialen Umsteuerung

Band 3

Konkurrenten oder Partner.

Ökobilanz undf Öko-Audit im Verlgeich

Band 4

Von der Vision zur Praxis.

Nachhaltiges Wirtschaften als Perspektive für Unternehmen

Band 5

Märkte ohne Grenzen.

Unternehmen im Spannungsfeld zwischen nachhaltigen Wirtschaften und globalen Wettbewerb

Band 6

Arbeit - Strategien der Existenzsicherung

Band 7

Mit neuer Energie in die Zukunft. Unternehmen zwischen Billigstrom- und Öko-Angeboten

Erhältlich bei: pan adress Semmelweisstraße 8, D-82152 Planegg

Fon $++49 /(0) 89 / 85709-155$, Fax -131

E-Mail kontakt@oekom.de, www.oekom.de 
(c) 20I0 Authors; licensee IÖW and oekom verlag. This is an article distributed under the terms of the Creative Commons Attribution Non-Commercial No Derivates License (http://creativecommons.org/licenses/by-nc-nd/3.o/), which permits unrestricted use, distribution, and reproduction in any medium, provided the original work is properly cited. 